\title{
Determination of Flow Profiles in Porous Media Using Shifts in Gamma Spectra
}

\begin{abstract}
A new technique was developed to determine the tracer location and fluid velocity in porous media nonintrusively. This technique exploits the competitive effects between the photoelectric interaction and the Compton scattering phenomenon to determine the distance between a radioactive tracer in a porous medium and an externally positioned detector. The photon energy distribution shifts toward lower photon energies as the tracer moves away from the detector. The shift in the energy distribution can be quantified by the ratio of the scattered photon intensity to the full energy photon intensity. A convective-dispersion model was used to determine the spatial distribution of the radioactive tracer. An analog Monte Carlo method was developed to simulate photon transport in porous media. Comparison between experimental data and the model shows that in-situ tracer velocities can be accurately predicted.
\end{abstract}

\author{
Jay K. Jasti \\ H. Scott Fogler \\ Department of Chemical Engineering \\ University of Michigan \\ Ann Arbor, Ml 48109
}

\section{Introduction}

Radioactive tracers are routinely used to determine in-situ velocity and mixing profiles in many industrial processes. Tracers have been used for a long time in medical research to detect tumors and to determine the size of internal organs (Koral and Johnston, 1974). Recently, gamma-ray-emitting tracers have been used to determine solid circulation patterns in fluidized beds (Lin et al., 1985). Gamma-ray-emitting tracers have been used for these applications because the photons possess large mean free paths in dense media and measurements can be made nonintrusively. This study focuses on the use of gamma radiation to determine $i n$-situ velocity profiles in porous media. It finds direct application in tracer logging where measurement of velocity profiles in the porous matrix surrounding the well bore is currently not possible.

Radioactive tracers are used to determine fluid flow patterns inside the wells by measuring the tracer transit time between two gamma-ray detectors separated by a known distance. These procedures, however, cannot identify the horizontal continuity of fluid flow in the porous matrix surrounding the well bore. The porous region surrounding an injection well, which is also called the skin zone, experiences changes in its flow conductivity due to several factors. Some of the factors contributing to these changes are: hydraulic fracturing, acid stimulation (Hoefner and Fogler, 1988), polymer injection, paraffin deposition, sand production, fines migration (Khilar and Fogler, 1987), emulsion blocking, and clay swelling (McKinley, 1985). The skin zone contributes the most to the flow resistance in an oil reservoir owing to the radial geometry where all of the flow goes through a point source or a sink. The proposed method uses the attenuation properties of gamma radiation to determine in-situ velocity profiles in the skin zone by monitoring the gamma energy distribution as the tracer moves away from an array of detectors situated in the well bore. Hence, it can be used to detect flow channeling, location and direction of propagation of fractures in the porous matrix surrounding the well bore.

This paper briefly describes interactions of gamma radiation with matter and an analog Monte Carlo method developed for simulating photon transport in porous media. A model used for predicting the spatial distribution of the radioactive tracer is discussed subsequently. Finally, a comparison of model predictions with experimental results is presented

\section{Interaction of Gamma Rays with Matter}

The transport of gamma rays in any given medium is governed by four principal interactions: photoelectric effect, Compton scattering, Rayleigh scattering, and pair production. Pair production interaction is important only when the energy of the photon exceeds $5.0 \mathrm{MeV}$. Since the isotopes used in this study emit photons with energy lower than $1.5 \mathrm{MeV}$, this interaction is not considered. Rayleigh scattering is negligible since it is dominated by the photoelectric effect. As a consequence, only two interactions are important for the photon energies used in this study. 


\section{Photoelectric effect}

During this process, the photon transfers all of its energy to one of the atomic electrons of the medium, ejecting it from the atom. The vacancy created by the ejected electron will be quickly filled by the rearrangement of electrons from the surroundings. The attenuation coefficient $(\tau)$ due to this process has the following dependence on the photon energy $(E)$ and the atomic number $(Z)$ of the medium (Knoll, 1979).

$$
\tau=\frac{C_{1} \rho Z^{n^{\prime}}}{E^{3}}
$$

The important characteristics of this interaction are the disappearance of the photon and its dominance at photon energies below $0.2 \mathrm{MeV}$.

\section{Compton scattering}

In this process, the incident photon interacts with an electron of the medium. The collision energy is shared between the recoil electron and the scattered secondary photon. Energy and momentum balances for this interaction give the following relation between the scattered photon energy $\left(E^{\prime}\right)$ and the incident photon energy $(E)$ (Knoll, 1979):

$$
E^{\prime}=\frac{E}{1+E \frac{(1-\cos \theta)}{m_{o} c^{2}}}
$$

where

$$
\begin{aligned}
\theta & =\text { angle of the direction of the scattered photon } \\
m_{o} & =\text { rest mass of the electron } \\
c & =\text { speed of light }
\end{aligned}
$$

The probability of a photon scattering in a direction $\theta, \theta+d \theta$ has been determined by Klein-Nishina (1929) using relativistic mechanics. The total cross section for Compton scattering is determined by integrating the Klein/Nishina function over all the angles. It has the following dependence on the properties of the medium:

$$
\sigma=0.75 S\left(E_{o}\right) C_{o} \rho N_{\text {avg }} \frac{Z}{A} g\left(E_{o}\right)
$$

The expressions $S\left(E_{o}\right)$ and $g\left(E_{o}\right)$ are well known and can be found in Knoll (1979). The first is the integral of the KleinNishina function over all the scatter angles and was derived assuming all the electrons are free. The function $g(E o)$ is a correction factor that incorporates the effect of the boundedness of the electrons. Compton scattering functions for various atoms at different scattering angles have been calculated by Cromer and Mann (1967) and Cromer (1969). For most of the elements in the periodic table, the value $Z / A$ is close to 0.5 . Hence, the value of $\sigma$ is virtually the same for all the elements and it is a function only of density. The principal characteristics of this interaction are the change of travel direction of the photon and the reduction of the photon energy.

\section{Cross Sections of Photon Interactions}

The relative importance of various gamma interactions with media can be quantified in terms of their cross-section values. If the flux of radiation at any point $x$ is $N(x)$, and $\mu$ is the cross-section value for an interaction, then the number of photons undergoing a given interaction in a path length $d x$ is

$$
d N=-N \mu d x
$$

The cross section, therefore, is equivalent to the probability of a photon undergoing an interaction within a unit distance. The negative sign is due to the fact that any interaction contributes in a net reduction in the photon fiux.

The total cross section for any medium is the sum of the individual cross sections representing various interactions such as: the photoelectric effect $\tau$, Compton scattering $\sigma$.

$$
\mu=\tau+\sigma
$$

The cross sections for any compound can be evaluated as the weighted sum of its elemental cross sections.

$$
\frac{\mu}{\rho}=\sum_{i} w_{i} \frac{\mu_{i}}{\rho_{i}}
$$

Elemental cross sections have been given for all of the elements by Storm and Israel (1970). Berea sandstone was used as the porous medium in this study and its cross sections were calculated using Eq. 6. The results are shown in Figure 1 in terms of the cross section as a function of the photon energy.

\section{Numerical Simulation of Photon Transport in Porous Media}

A Monte Carlo method was developed to simulate photon transport in porous media. The governing equation for predicting the photon distribution function $n(r, \Omega, E)$ is:

$$
\begin{aligned}
\Omega \cdot \nabla n(r, \Omega, E)+\mu(E) n(r, \Omega, E) & \\
=\int_{4 \pi} d \Omega^{\prime} \int d E^{\prime} \sigma\left(\Omega^{\prime} \rightarrow \Omega, E^{\prime} \rightarrow E\right) & n(r, \Omega, E) \\
& +S(r, \Omega, E)
\end{aligned}
$$

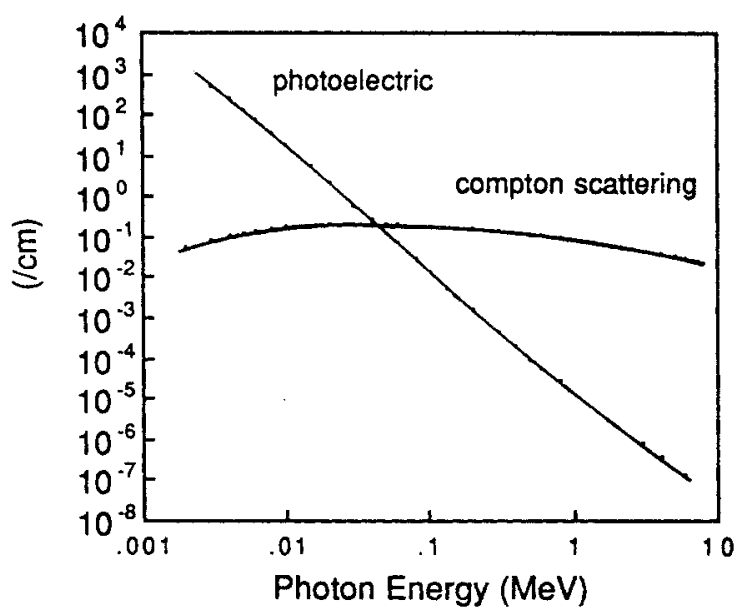

Figure 1. Photon cross sections for Berea sandstone. 
The distribution function $[n(r, \Omega, E) d r d \Omega d E]$ represents the number of photons in a unit volume $d r^{3}$ at position $r$, traveling in the direction $d \Omega$ about $\Omega$ and possessing the energy in the range $E$ to $E+d E$. The terms in the governing equation represent a balance between the rates of photon removal and addition in the control-phase space: $d r d \Omega d E$. The first term in the integral equation is the surface term representing the removal rate of photons from the control volume, i.e., the net change in the number of photons. The second term represents the number of photons lost from the control volume due to various interactions with the medium. The third term represents the addition of photons to the control volume due to scattering. The final term is the source term, which represents the addition of photons due to the presence of a photon source in the control volume.

Several techniques, such as analytical methods, moment methods, discrete ordinate methods, finite difference, finite element, and spectral and Monte Carlo methods, have been used to solve the transport equation (Duderstadt and Martin, 1979). Analytical methods require approximations to render the equation solvable. All the other techniques require enormous computing time since the solution is needed for six independent variables (e.g., three directions, two angles and energy). An analog Monte Carlo method was developed to simulate photon transport in porous media in this study and the details of the model are given below.

\section{Monte Carlo Method}

The spatial distribution of the photon source, the geometry of the system, and gamma cross sections of the media are provided as inputs to the model. Several photon histories are then calculated in a manner similar to the random walk model, and the photon energy distribution crossing the surface of interest is tallied. The inherent assumption is that photon transport is a linear process. It is a valid assumption since nonlinear, multiparticle phenomena occur at photon energies far exceeding the values used here. A flow chart illustrating the procedure used is shown in Figure 2 and various steps in the chart are briefly described below.

The location of photon release is determined first by sampling from a given spatial distribution function of the source. The source distribution function is determined by a tracer transport model. The direction of photon travel is sampled from a uniform distribution since the source emits photons isotropically. The distance the photon travels is sampled using the energy of the photon, the total cross section of the medium, and the exponential relation resulting from the integration of Eq. 4. The photon is then moved that distance in the direction sampled. At this point, the type of interaction is determined knowing the photon energy and the cross sections of the medium. The probability for an interaction to occur is simply the normalized cross section for that interaction. If the photoelectric effect is chosen, then the photon history is terminated and a new history is initiated from the beginning. If a scattering event is chosen, the direction of photon scatter and its new energy are sampled from the modified Klein-Nishina distribution. The history of the scattered photon is continued by sampling its distance of travel. The procedure is used several times and the photon energy distribution crossing a surface of interest is tallied. The sampling of the random numbers is made either by using the cumulative distribution method or by using one of the rejection techniques (Carter and Cashwell, 1975).

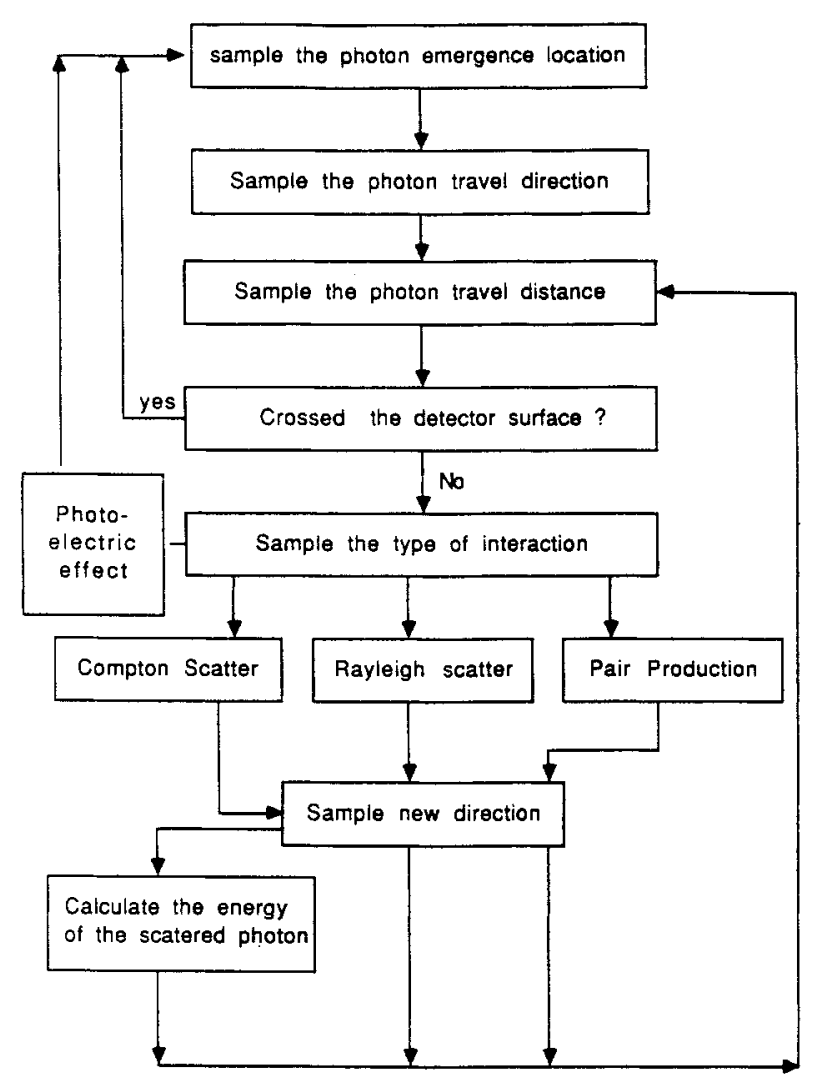

Figure 2. Monte Carlo procedures used for simulating photon transport.

The above procedures are used to generate photon histories $N_{\max }$ until the variance in the result is less than $15 \%$. Each history is terminated when one of the following events occur:

1. The photon undergoes the photoelectric effect.

2. The photon crosses the boundary of the system. The histories of the photons escaping the lead shield are not pursued since the probability of these photons reentering the system and reaching the region of interest is extremely low. This is a valid assumption for our system since a $5-\mathrm{cm}$ thick layer of lead was used as a shield in all the experiments.

3. The photon crosses the surface of the detector. The energy distribution of the photons crossing the surface is calculated by incrementing the numbe of photons within the energy band $E$, $E+d E$ by the weight function of the photon. The weight function of the photon will have a unit value if no biasing schemes are used, whereas it may have other values if any biasing schemes are used. The biasing schemes used are explained in the next section.

The central limit theorem is used as a basis for estimating statistical errors in the expectation value. For every photon crossing the surface of interest, the score corresponding to the photon energy is increased by the weight function of the photon. In addition, the sum of the weight squares is also tallied. The mean and the intrinsic variance of the result are then calculated using the following relations:

$$
\bar{N}(E)=\frac{1}{N_{\max }} \sum_{1}^{n} w_{i}
$$




$$
\sigma^{2}(E)=\frac{1}{\left(N_{\max }-1\right)}\left[\sum_{1}^{n} w_{i}^{2}-N_{\max } \bar{N}(E)^{2}\right]
$$

where $w_{i}$ is the weight function of the photon.

\section{Photon splitting and Russian roulette}

Photon splitting and Russian roulette improve the efficiency of the Monte Carlo method by artificially biasing the calculations to emphasize the photons moving toward the surface of interest. This procedure is illustrated in Figure 3. The splitting procedure is implemented by doubling the number of photons when they cross an imaginary plane called the splitting plane. In order to avoid biasing the results, the weight of each photon is reduced by half when it crosses the splitting plane. The histories of the photons resulting from splitting are then pursued independently. In this example, the splitting plane is denoted as $S_{2}$; and for the purposes of illustration, the photon attenuation between the planes $S_{i}$ is assumed to be 0.1 . The photon source is located on the $S_{3}$ plane, and $S_{1}$ represents the plane of interest. In other words, the fraction of the photons crossing the $S_{1}$ plane need to be calculated. This fraction is called the expectation value. When no biasing schemes are used, a minimum of 100 photon histories is required to get an expectation value of 0.01 . A smaller number of photon histories, however, is needed to obtain the same expectation value when photon splitting is implemented. In this example, the same result can be obtained with only 50 histories. In this case, only five photons reach the $S_{2}$ plane since attenuation between the planes is 0.1 . The number is doubled and ten photon histories are performed between $S_{2}$ and $S_{1}$ plane, and the weight function of each photon will be reduced to 0.5 . Russian roulette is similar to the splitting procedure, except that it is used to reduce the number of photon histories moving toward the regions of lesser importance. The number of photons crossing a plane is reduced by half and their weights are doubled. In this example, this procedure would be implemented

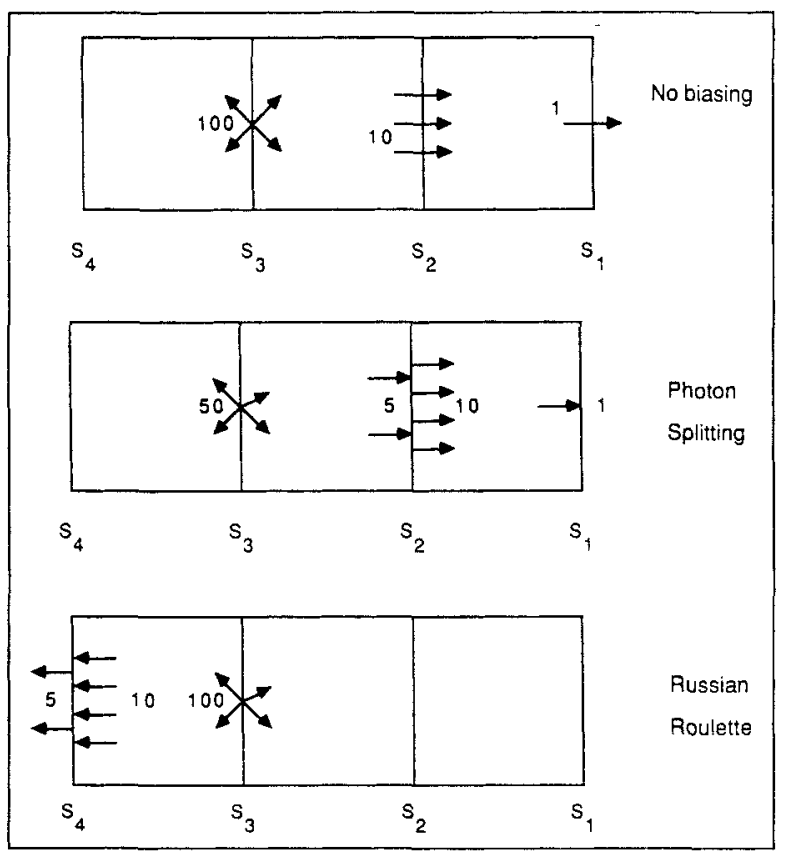

Figure 3. Biasing schemes: Splitting \& Russian roulette. at the $S_{4}$ plane. In other words, when a photon crosses the $S_{4}$ plane in the opposite direction, it is allowed to survive with a probability of 0.5 .

The factors 2 and 0.5 are chosen arbitrarily to explain the procedures. Any number of planes and any factor $M$ can be used for biasing. For example, a photon crossing a splitting plane can be divided into $M$ photons each with a weight of $1 / M$. The distance between the splitting planes, the number of planes, and the splitting value are generally set by an optimization procedure. A detailed discussion of these procedures can be found in Lux (1983), Booth (1985), and Dubi and Goldfeld (1985). In this study, the splitting planes were separated by the mean free path of the full energy photon and a splitting factor of 2 was used.

\section{Gamma Transport in Porous Media}

The Monte Carlo method was used to simulate photon transport in an infinite Berea sandstone medium. A point source emitting $0.365 \mathrm{MeV}$ photons was used for the simulation and the photon energy distributions crossing spherical planes at various distances from the source were calculated. The photon energy distribution crossing a spherical surface a distance $6 \mathrm{~cm}$ from the source is shown in Figure 4. The ordinate in Figure 4 represents the number of photons crossing a spherical plane within the energy region $E$ to $E+d E$ for $N_{\max }$ photon histories. The maximum standard deviation of the result is about $5 \%$. The energy distribution shown in Figure 4 has two distinct features. The Dirac delta at $0.365 \mathrm{MeV}$ represent the number of photons crossing the spherical plane without undergoing any interaction in the medium; these are called the full energy photons. The second feature of the spectrum is the continuum of photons having energy less than $0.365 \mathrm{MeV}$. These photons have undergone one or more scattering interactions in the medium, and the area under the continuum represents the total number of scattered photons reaching the detector.

The effect of travel distance on the scattered photon energy distribution is shown in Figures 5 and 6. At shorter distances, the scattered photon energy distribution would be the same as the Klein-Nishina distribution which represents the distribution of photons undergoing one Compton scatter event. As the distance is increased, the scattered photons undergo further scattering and their energy distribution shifts to lower energies.

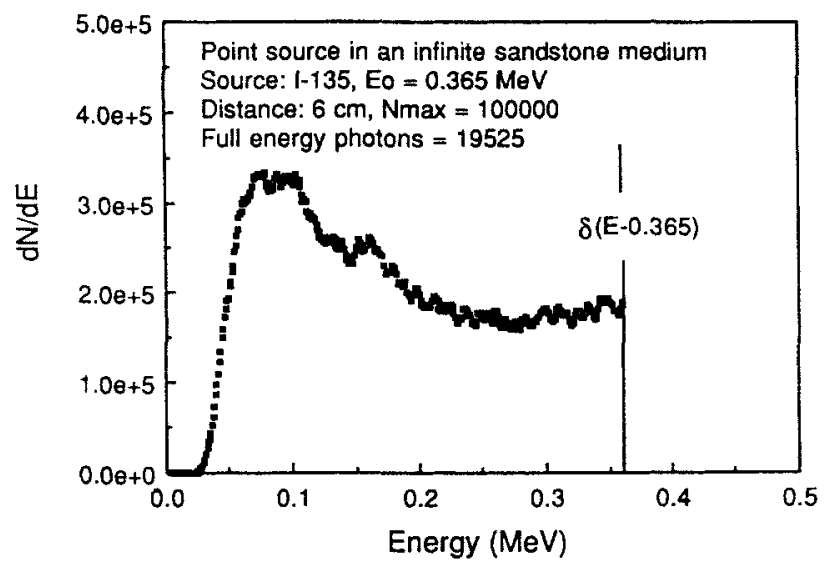

Figure 4. Photon energy distribution calculated by the Monte Carlo method. 


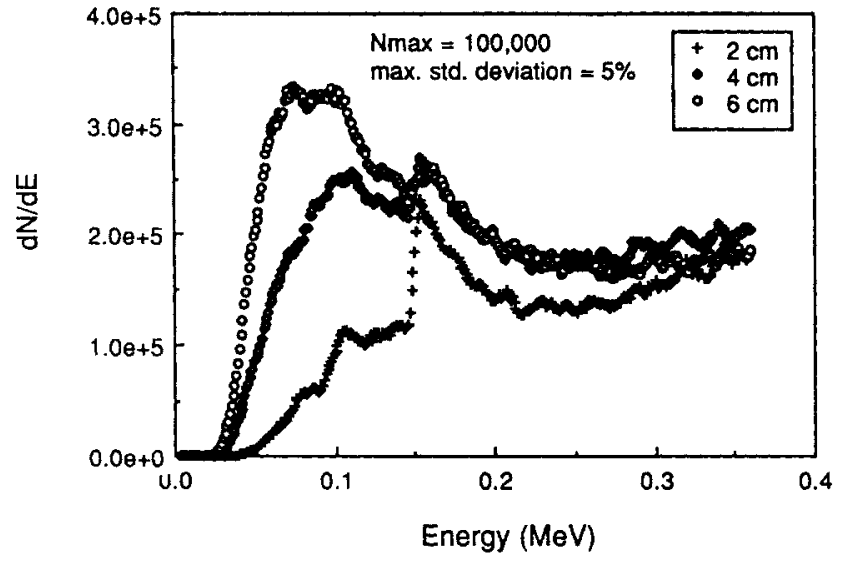

Figure 5. Effect of photon travel distance on the scattered photon energy distribution at short distances as predicted by the Monte Carlo method.

If the distance is increased further, the shift in the energy distribution no longer occurs (Figure 6). This is attributed to the dominance of the photoelectric effect at lower photon energies. The intensity of the scattered photon energy distribution decays with increasing distances since the photoelectric effect is an absorptive interaction. This can be seen as the reduction in the area under the curves shown in Figure 6.

The attenuation of the full energy photons and the scattered photons with increasing distance between the detector and the source is shown in Figure 7. Here, the scttered fraction is defined as the area under the curves in Figures 5 and 6 normalized by $N_{\max }$. The full energy fraction decreases in an exponential manner with increasing distance. The slope of this curve is simply the product of the cross section and the density of the material. On the other hand, the scattered fraction initially increases with distance, reaches a maximum, and then decreases. As explained earlier, this maximum is due to the competition between multiple Compton scattering and the photoelectric interaction. The information in Figure 7 can be used to determined the location of the photon source in the porous medium. However, one can only measure the full energy

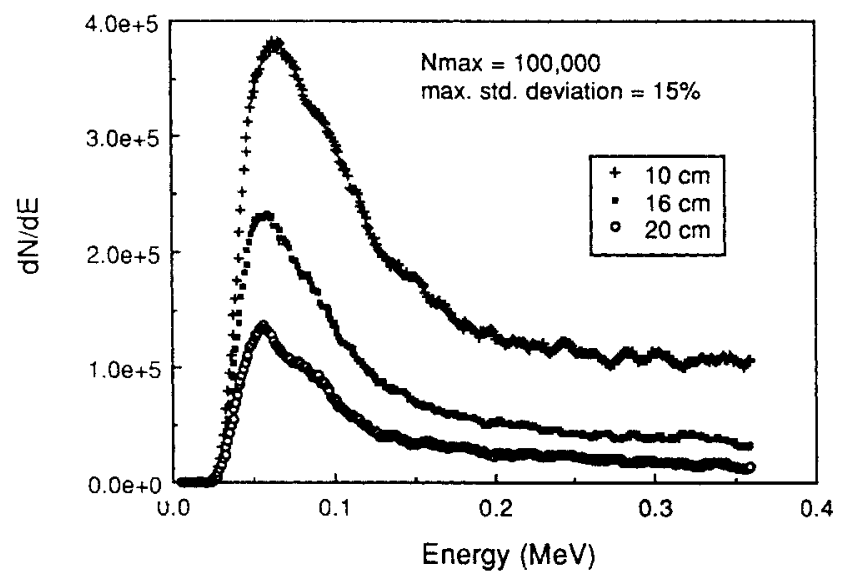

Figure 6. Effect of photon travel distance on the scattered photon energy distribution at long distances as predicted by the Monte Carlo method.

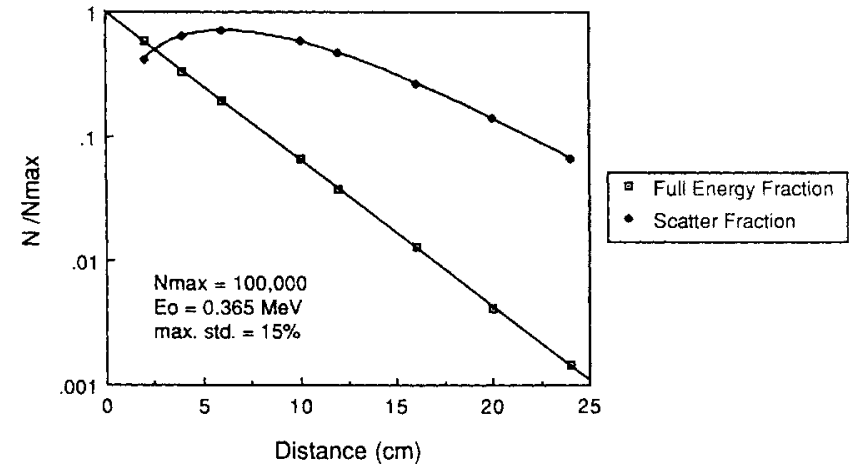

Figure 7. Attenuation of gamma radiation in Berea sandstone as predicted by the Monte Carlo method.

intensity and the scattered intensity reaching the detector. The normalizing factor in Figure 7, namely the activity of tracer in each zone, is an unknown quantity in a full-scale system. Consequently, a parameter which is independent of the activity of the tracer needs to be used to locate the tracer. The rati of the scattered photon intensity to the full energy photon intensity (scatter to peak ratio) is independent of the tracer activity. Figure 7 reveals that the full energy intensity decays faster than the scattered intensity; and as a result, the scatter to full peak intensity ratio will increase monotonically with the distance and therefore can be used to determine the location of the tracer.

\section{Tracer Transport in Porous Media}

The above analysis was performed for an isotropic porous medium with a point source. In reality, a tracer pulse injected into a porous medium will distribute itself due to dispersion. It is well known that the spreading of the tracer in a porous medium can be approximated by an effective dispersion coefficient and that the standard deviation of the tracer spatial distribution increases with the square root of time as the tracer pulse moves through the porous matrix (Blackwell, 1985).

$$
\overline{\sigma^{2}}=2 D t
$$

The movement of a nonabsorbing tracer in a porous medium can be represented by the convective-dispersion equation (Perkins and Johnston, 1963).

$$
\frac{\partial \xi}{\partial \Theta}=\frac{1}{P e} \frac{\partial^{2} \xi}{\partial Z^{\prime 2}}-\frac{\partial \xi}{\partial Z^{\prime}}
$$

The following initial and boundary conditions have been used:

$$
\begin{gathered}
\xi-\frac{1}{P_{e}} \frac{\partial \xi}{\partial Z^{\prime}}=\xi_{o}(\Theta) \text { at } Z^{\prime}=0 \\
\frac{\partial \xi}{\partial Z^{\prime}}=0 \text { at } Z^{\prime}=1 \\
\xi\left(Z^{\prime}\right)=0 \text { at } \Theta=0 \\
\xi_{o}=1 \text { when } 0<\Theta \leq \Theta_{1} \\
\xi_{0}=0 \text { when } \Theta>\Theta_{1}
\end{gathered}
$$


The value $\Theta_{1}$ represents the pore volumes of tracer injected into the porous medium. The above system of equations can be solved analytically using a Laplace transform. The solution was provided as an input to the gamma transport code. The intensity of gamma radiation attenuates as the tracer moves away from the detector as shown in Figure 8. The results reveal that the attenuation rates measured on a pore volume basis are independent of tracer velocity in the porous medium. The principal reason for the invariance is the weak nonlinear dependence of dispersion coefficient on the interstitial velocity in the convection regime. The following expression was found to be valid for all the naturally occurring consolidated porous media (Fried and Cambarnus, 1971).

$$
D=\alpha v^{n}
$$

The exponent in the above equation is in the range: 1.0-1.2. As a consequence, the Peclet number $(P e=v L / D)$ in Eq. 11 is weakly dependent on the velocity. The important conclusion from this result is that the tracer velocity can be determined from the time rate of change of gamma intensity.

\section{Effect of Tailing of the Tracer}

Tailing is another phenomenon which occurs along with dispersion during the movement of a tracer through a porous medium. Tailing of the tracer molecules is caused by the dead zones in the porous medium where there is no direct flow. Transport of the tracer between the dead zones and the bulk pore space is controlled by molecular diffusion. As a consequence, some tracer molecules lag behind the bulk tracer pulse and the spatial distribution of the tracer assumes the shape of a skewed Gaussian function. This capacitance phenomenon could be detrimental to the technique proposed here since a small amount of tracer lagging behind could contribute more gamma intensity to the detector than the bulk tracer slug owing to its proximity to the detector. The bulk tracer pulse may not contribute significantly since gamma intensity attenuates exponentially with distance.

The regions of pore space with no direct flow and the pore spaces with extremely large resistances to flow contribute to the

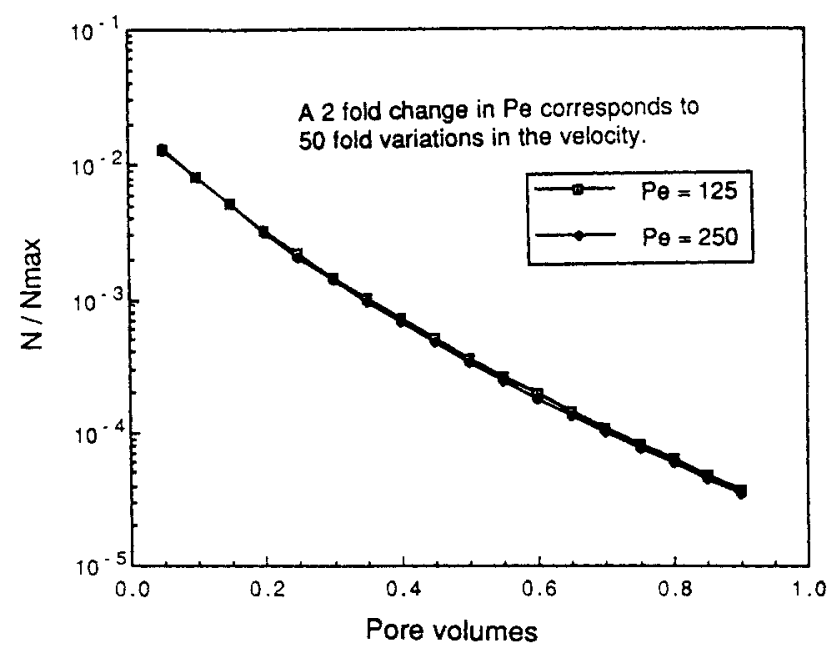

Figure 8. Effect of the Peclet number on the attenuation of full energy intensity. tailing of the tracer. The effect of microscopic dead zones on the movement of a tracer pulse in a porous medium has been studied in detail and the results were presented by Jasti et al. (1988). Our principal conclusion was that the model developed by Coats and Smith (1964) can be used to represent capacitance phenomena at the microscopic scale. However, the three parameters used for the model are not adjustable and should be determined independently. The procedures for determining these parameters, the Peclet number $(P e)$, the Damkohler number for mass transfer $(D a)$, and the fraction of dead-end pore space $(1-F)$ from experimental data are given by Jasti et al. (1988). The effect of tailing on gamma transport is studied here by coupling our Monte Carlo simulation with the Coats and Smith model.

The governing equations for the movement of a tracer slug in a porous medium are:

$$
\begin{gathered}
(1-F) \frac{\partial \psi}{\partial \Theta}+F \frac{\partial \xi}{\partial \Theta}=\frac{1}{P e} \frac{\partial^{2} \xi}{\partial Z^{\prime 2}}-\frac{\partial \xi}{\partial Z^{\prime}} \\
(1-F) \frac{\partial \psi}{\partial \Theta}=D a(\xi-\psi)
\end{gathered}
$$

The boundary conditions for the above system of equations are the same as Eqs. 12-16. The tracer concentration profile was calculated using the analytical solution to Eqs. 18 and 19 (Baker, 1977), and the solutions were provided as an input to the Monte Carlo code. The attenuation rates for the full energy intensity were calculated for different dead end fractions and the results are shown in Figure 9. At short times $[\Theta<(1-F) / D a]$, the attenuation rates for all the cases are nearly the same since the capacitance phenomenon manifests itself at longer times. Large deviations from the base case occur when the dead end pore fraction exceeds $30 \%$ of the total pore space. Since most of the naturally occurring porous media such as sandstones and carbonates contain less than $15 \%$ dead-end pore space, we conclude from this study that the tailing phenomenon does not influence gamma attenuation rates.

\section{Experimental Results}

Experiments were carried out using gamma-ray-emitting tracers and Berea sandstone cores, $2.5 \mathrm{~cm}$ in diameter and $22 \mathrm{~cm}$ in length, to establish the validity of the model. The porous

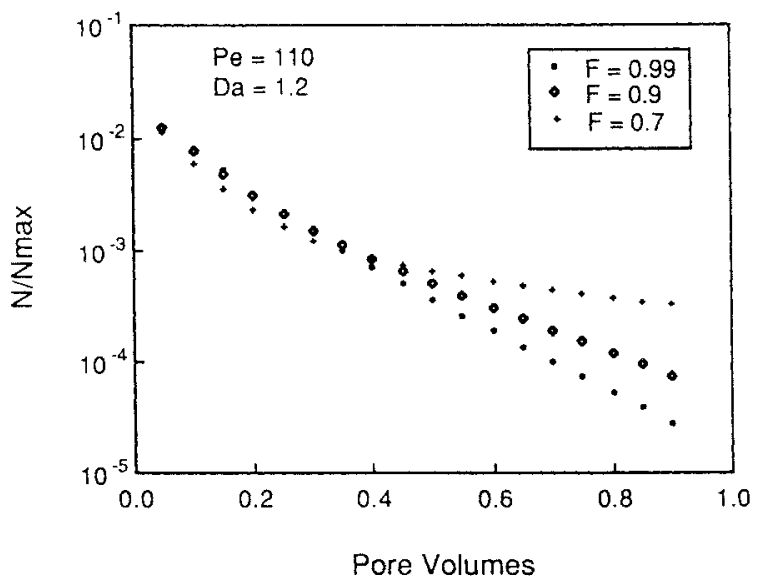

Figure 9. Effect of flowing fraction on the attenuation of full energy intensity. 
matrix was sealed into an aluminum pipe using white epoxy resin and the ends were closed by threaded distributor caps to allow uniform injection and removal of fluids. The experimental system is shown in Figure 10. The core was surrounded by silica bricks having a thickness of $15 \mathrm{~cm}$ and a lead shield having a thickness of $5 \mathrm{~cm}$. Silica bricks simulate a sandstone system since the elemental compositions are nearly the same. Rubidium86 chloride $(1.076 \mathrm{MeV})$ was used as the tracer, whereas magnesium in the form of magnesium chloride was used as the eluting ion to minimize ion exchange.

At the onset of the experiment, 0.02 pore volumes of the tracer with an activity of 240 microcuries were injected into the porous medium. A sodium iodide scintillation detector (7.5 $\mathrm{cm} \times 7.5 \mathrm{~cm}$ ) was positioned near the inlet face of the core, and gamma spectra were recorded as the tracer pulse moved away from the detector. Data acquisition was made using an ND-7600 data processing system. Figures 11 and 12 show typical gamma energy spectra recorded during an experiment and model predictions for this experimental system. The photon energy distribution incident upon the detector is different from the energy distribution displayed by the detector. This distortion of the energy distribution is attributed to the poor resolution and partial photon energy absorption in the detector. The detector efficiency and its resolution function have been experimentally determined using several standard gamma sources. Monte Carlo predictions of photon energy distributions have been convoluted with the detector response functions in order to account for the distortions caused by the detector. Figures 11 and 12 show that the model accurately simulates transport of gamma radiation. Some discrepancy at lower photon energies indicate that the photoelectric cross sections for the medium are larger than the values calculated using Eq. 6.

Each of the photon energy spectra has been reduced to three parameters: the area under the full energy peak, the area under the rest of the energy spectrum, and the ratio of these areas. These parameters are called the full energy intensity, the scattered photon intensity, and the scatter to full peak intensity ratio, respectively. Experimental data obtained at two flow rates are presented in Figures 13 and 14. Photon intensity measurements beyond 0.85 pore volumes should be neglected since the tracer pulse begins to exit the experimental system. The results clearly show that the data are independent of the flow rate when plotted as a function of the pore volumes; and they confirm the model predictions. Since the ordinate is made nondimensional using the velocity, the time rate of change of these parameters will be proportional to the velocity.

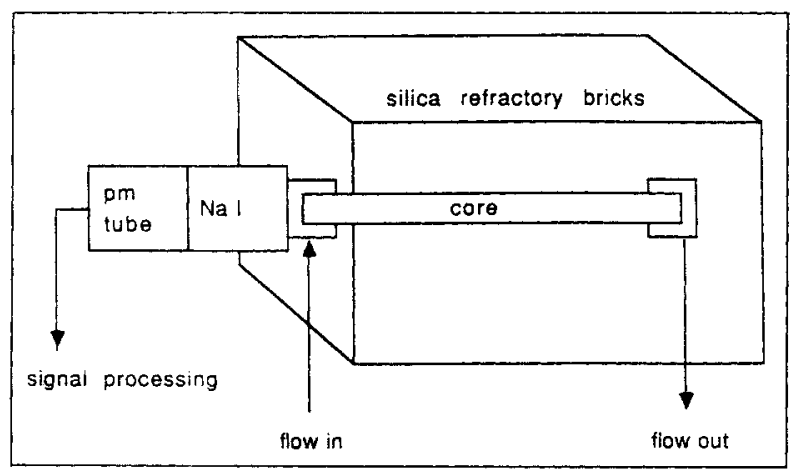

Figure 10. Experimental system.

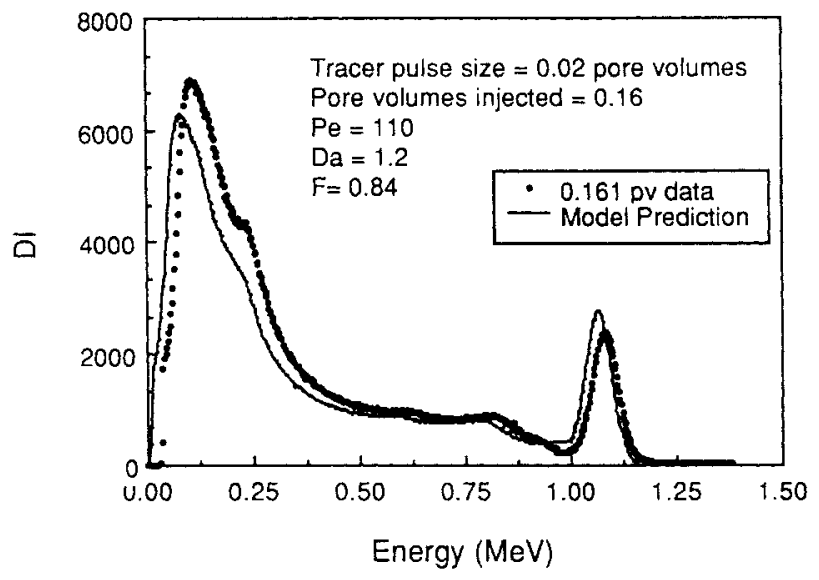

Figure 11. Model predictions vs. experimental gamma energy spectra at short times.

\section{Determination of in-situ Velocity Profiles in Porous Media}

The attenuation rate of full energy gamma intensity and scattered gamma intensity was found to be proportional to the tracer velocity in the porous matrix. The rates of gamma attenuation, however, also depend on the orientation of the detector, the geometry of the system, and the cross sections of the medium. An understanding of how these parameters influence the decay rates of gamma intensity is required, since, for the full-scale system, only time-dependent data will be available instead of information on a pore volume basis.

A schematic representation of the procedure for solving the inverse problem is shown in Figure 15. The time-dependent experimental data need to be convoluted with distancedependent information in order to determine the tracer velocity. The spatially-dependent data can be obtained using either a model or a pilot system which has the same geometry, detector orientation, medium properties and spatial distribution of the source as the full-scale system. A model would make the convolution easier, and the Monte Carlo method, in conjunction with the tracer transport model, has already predicted gamma transport in experimental systems very accurately. The tracer

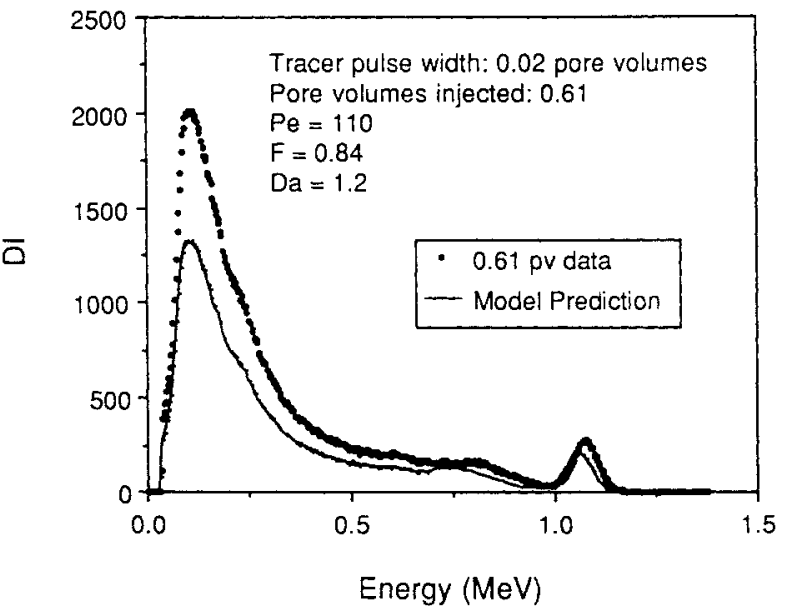

Figure 12. Model predictions vs. experimental data at long times. 


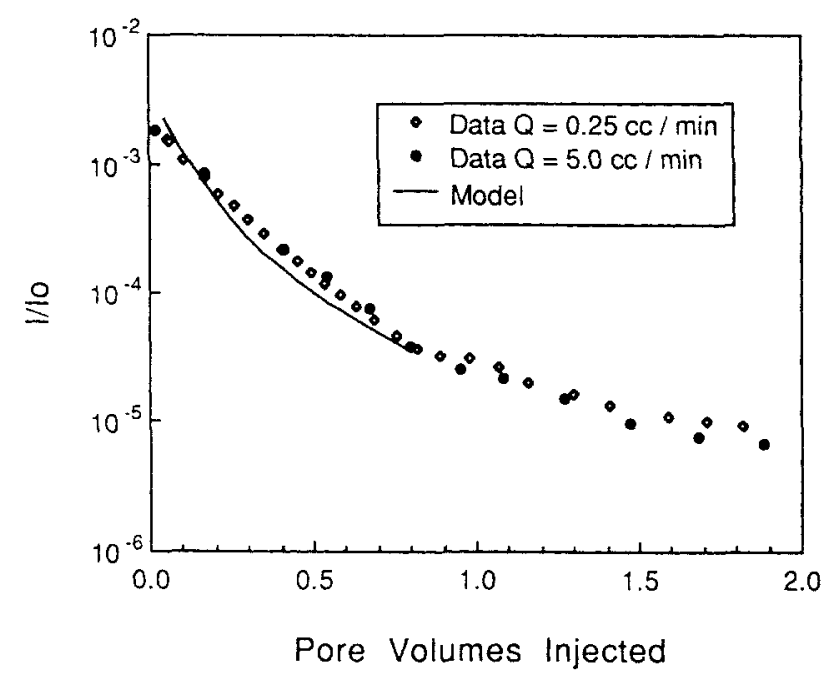

Figure 13. Effect of flow rate on the attenuation of full energy intensity

velocity in a porous medium can be determined from one of the following relations:

$$
\begin{gathered}
v=\frac{d R}{d t_{\text {expt }}} \frac{d x}{d R_{\text {model }}} \\
v=\frac{d(I / I o)}{d t_{\text {expt }}} \frac{d x}{d(I / I o)_{\text {model }}}
\end{gathered}
$$

where

$R=$ ratio of the scattered gamma intensity to the full-energy intensity

$t=$ time

$x=$ location of the mode of the tracer distribution

$I=$ full-energy photon intensity recorded by the detector

$I O=$ total number of full-energy photons released by the detector

$v=$ interstitial velocity

The slopes of the curves shown in Figure 15 vary with the abscissa. For this reason, the derivatives in Eq. 20 or 21 should be evaluated at the same $R$ or $I / I O$ values. However, the activity Io of the tracer in the full-scale system is an unknown quantity and one can only measure the gamma intensity $I$. Consequently, the derivatives can be evaluated only at the same $R$.

Another important criterion is the choice of the model used for determining the derivatives in Eq. 20. Figure 9 shows that the capacitance effects are negligible at short separation distances between the detector and the source and that they are only important beyond 0.3 pore volumes. The velocities were calculated using both dispersion and capacitance models and compared in Figures 16 and 17 with actual velocities used in the experiments. At shorter distances between the tracer and the detector, the predictions of both dispersion and capacitance models are identical and the calculated velocities are within $5 \%$ of the actual values. At longer separation distances between the tracer and the detector, the capacitance model predicts the location of the tracer more accurately than the dispersion model.

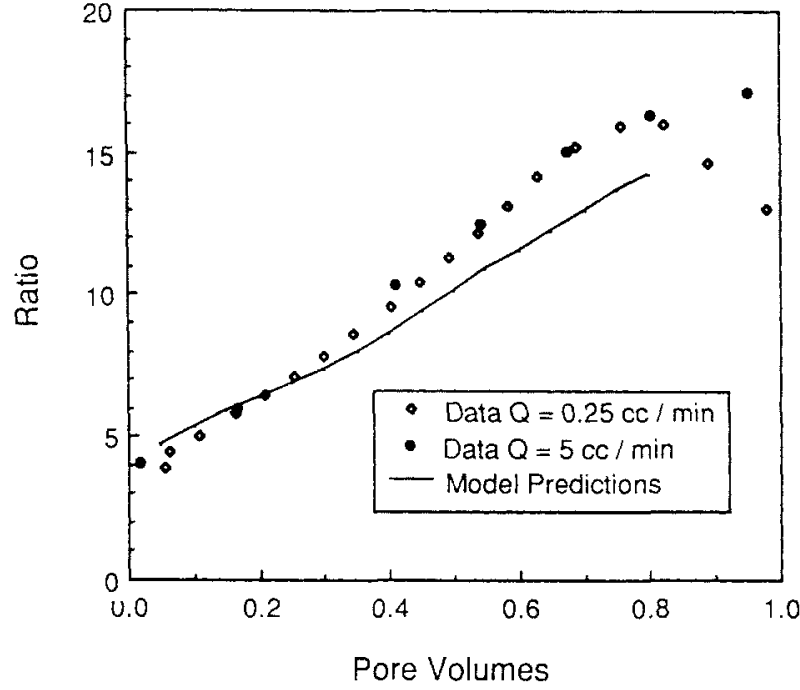

Figure 14. Effect of flow rate on the ratio of scattered photon intensity to the full energy photon intensity.

At higher flow rates, the dispersion model underpredicts the velocity by $35 \%$. Nevertheless, the dispersion model is recommended for the full-scale application, since it is accurate at lower velocities and only requires one parameter, whereas the capacitance model requires three parameters. Correlations are

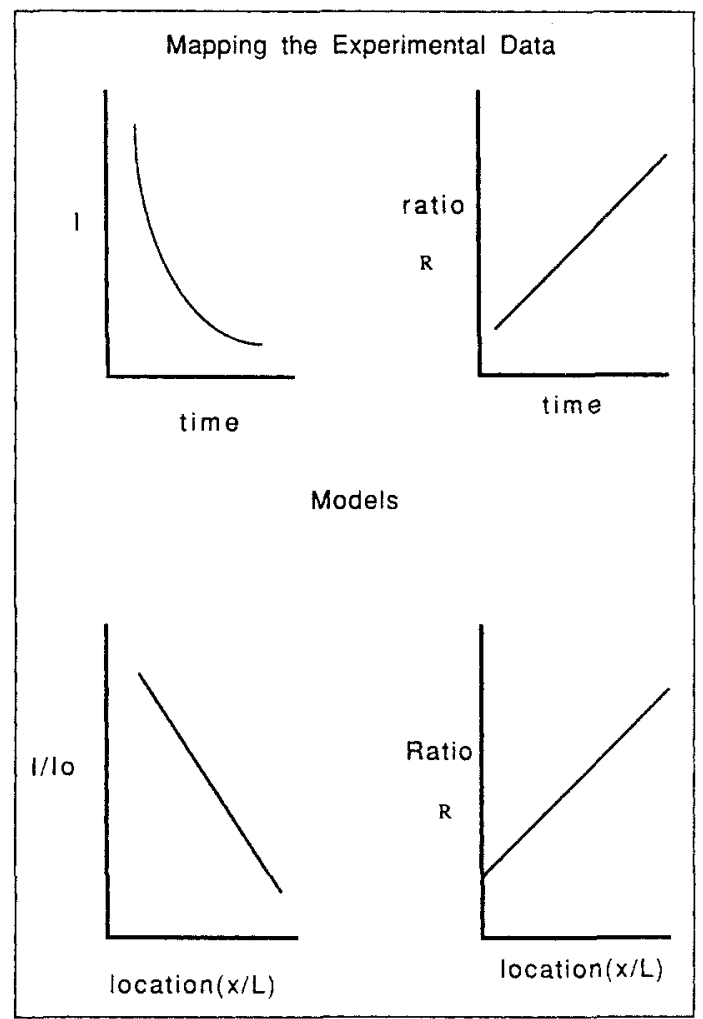

Figure 15. Mapping required to determine in-situ velocity using time-dependent experimental data and model predictions. 


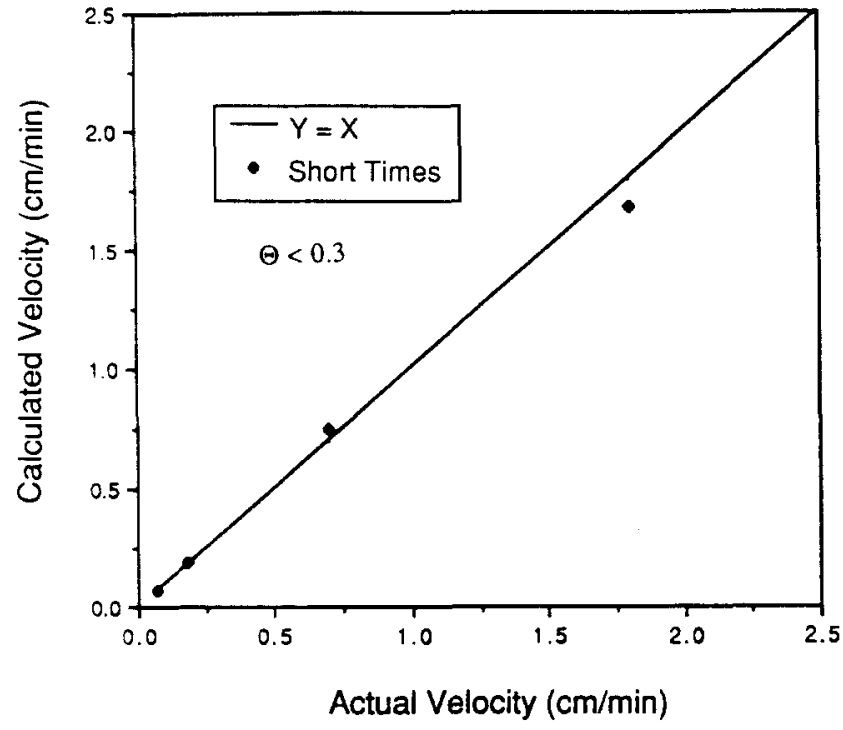

Figure 16. Model prediction of in-situ velocity v8. experimental data at short times.

available in the literature for estimating the dispersion coefficients in porous media (Blackwell, 1985).

The technique proposed here has some limitations when applied to a full-scale three-dimensional system. A detailed prediction of tracer spatial distribution in a three-dimensional system would require photon intensity data at various orientations between the source and the detector, and the use of tomographic reconstruction procedures. Unfortunately, the movement of the detector in a full-scale system is restricted to the vertical direction in a well bore. It is possible to detect vertical movement of the tracer by using an array of detectors spaced in the vertical direction and measuring time-delayed responses among the detectors. Average horizontal velocities can be determined by measuring the decay rate of gamma

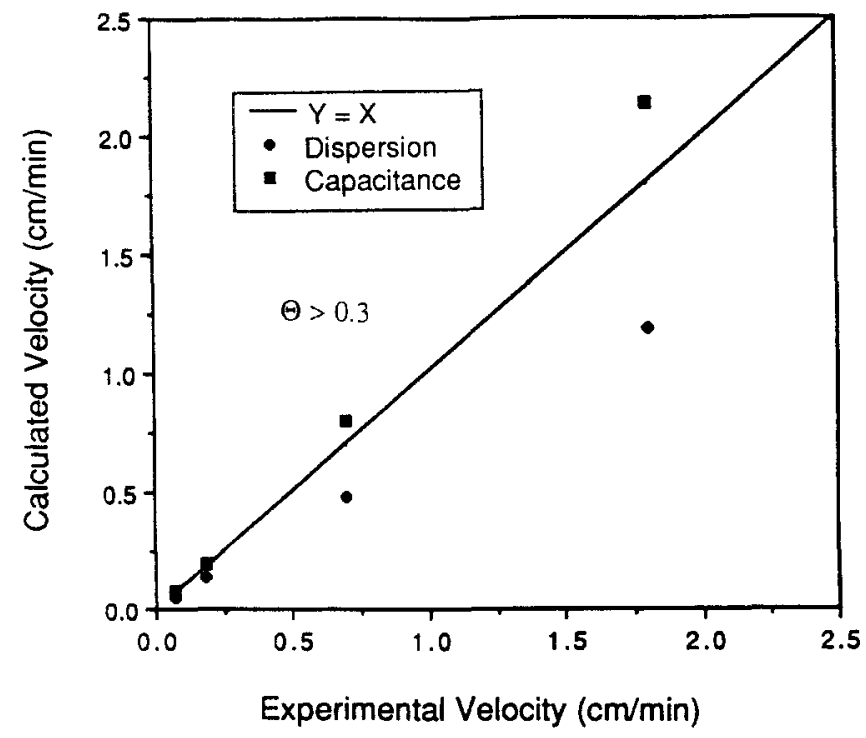

Figure 17. Model prediction of In-situ velocity vs. experimental data at longer times. intensity values. It is conceivable to measure the horizontal velocity components using shielded gamma detectors.

\section{Conclusions}

- The concept of using the shift in gamma energy distribution to determine the location of the radioactive source has been demonstrated. The changes in the photon energy distribution can be quantified using the ratio of the scattered photon intensity to the full energy photon intensity.

- The ratio of scattered photon intensity to the full energy photon intensity is independent of the amount of tracer present in a porous medium. Therefore, the location of the tracer can be determined without the knowledge of the amount of tracer present in a porous region.

- In-situ tracer velocities in the porous matrix can be determined by measuring the attenuation rates of either the full energy or the scattered gamma intensity.

- The spreading of tracer in a porous medium by dispersion and tailing has negligible effect on the measurement of tracer velocity. This is attributed to the weak dependence of the Peclet number on the interstitial velocity.

- A Monte Carlo model in conjunction with a tracer transport model can be used to simulate gamma transport in porous media accurately.

\section{Notation}

$$
\begin{aligned}
A & =\text { atomic weight } \\
C o & =\text { proportionality constant in Eq. } 3 \\
C 1 & =\text { constant in Eq. } 1 \\
D & =\text { dispersion coefficient, } \mathrm{cm}^{2} / \mathrm{s} \\
D a & =\text { Damkohler number }
\end{aligned}
$$

$D I=$ number of photons recorded by the detector in the range $E$, $E+d E$

$E=$ photon energy, $\mathrm{MeV}$

$E^{\prime}=$ scattered photon energy, $\mathrm{MeV}$

$F=$ flowing fraction of the pore space

$I=$ number of photons recorded by the detector in a unit time, No./min

$I o=$ number of photons emitted by the tracer in a unit time, No./min

$L=$ length of the porous medium, $\mathrm{cm}$

$m_{o} c^{2}=$ constant, $0.511 \mathrm{MeV}$

$M=$ splitting factor

$N=$ photon flux reaching the plane of interest

$n=$ photon distribution function

$n^{\prime}=$ value between 3 and 4 in Eq. 1

$N_{\text {avg }}=$ Avagadro number

$N_{\max }=$ number of random walks performed in a simulator.

$N(E)=$ number of photons reaching the plane of interest with energy E

$P e=$ Peclet number $(v L / D)$

$r=$ distance, $\mathrm{cm}$

$S=$ spatial distribution of a radioactive tracer

$S(E o)=$ Compton scattering function

$g(E o)=$ correction factor for the Compton scattering function

$t=$ time, $\mathrm{s}$

$v=$ velocity, $\mathrm{cm} / \mathrm{s}$

$w_{i}=$ weight fraction

$x=$ distance, $\mathrm{cm}$

$Z=$ atomic number

$Z^{\prime}=$ dimensionless distance

\section{Greek letters}

$\sigma=$ cross section for Compton scattering, cm

$\tau=$ cross section for the photon electric effect, $\mathrm{cm}$

$\rho=$ density of the porous medium, $\mathrm{g} / \mathrm{cm}^{3}$ 
$\theta=$ angle of photon scatter

$\boldsymbol{\theta}=$ dimensionless time (pore volumes of fluid injected)

$\Theta_{1}=$ pore volumes of tracer injected

$\mu=$ total cross section, $\mathrm{cm}$

$\Omega=$ direction of photon travel

$\sigma^{2}=$ variance of a function

$\xi=$ nondimensional tracer concentration in the flowing pore space

$\Psi=$ nondimensional tracer concentration in the dead-end pore space

\section{Literature Cited}

Baker, L.E., "Effects of Dispersion and Dead-End Pore Volume in Miscible Flooding," Soc. Pet. Eng. J., 219 (June, 1977).

Booth, T. E., "Monte Carlo Variance Comparison for Expected Value vs. Sampled Splitting," Nucl. Sci. and Eng., 89(4), 305 (1985).

Blackwell, R. J., "Miscible Processes II," SPE Reprint Series, Soc. of Petrol. Engrs., Richardson, TX (1985).

Carter, L. L., and E. D. Cashwell, "Particle Transport Simulation with the Monte Carlo Method," Technical Information Center, U.S.E.R.D.A., TID-26607 (1975).

Cromer, D. T., and J. B. Mann, "Compton Scattering Factors for Spherically Symmetric Free Atoms," J. Chem. Phys. 47(6), 1892 (1967).

Cromer, D. T., "Compton Scattering Factors for Aspherical Free Atoms," J. Chem. Phys., 50(1), 4857 (1969).

Coats, K. H., and B. D. Smith, "Dead-End Pore Volume and Dispersion in Porous Media," SPEJ, 73 (Mar., 1964).

Dubi, A., and A. Goldfeld, "Application of the Single Surface Extended Model of Geometrical Splitting in Monte Carlo," Nucl. Sci. \& Eng., 91(4), 470 (1985)

Duderstadt, J. J., and W. Martin, Transport Theory, Wiley, New York (1979).

Fried, J. J., and M. A. Cambarnus, "Dispersion in porous media," $A d v$. in Hydrosci, 7, 169 (1971).
Hoefner, M. L., and H. S. Fogler., "Pore Evolution and Channel Formation During Flow and Reaction in Porous Media," AIChE J. 34(1), 45 (1988).

Jasti, J., "Use of Radioactive Tracers to Predict Velocity Profiles Near Injection Wells," PhD Diss. Univ. of Michigan, Ann Arbor (1988).

Jasti, J. K., R. N. Vaidya, and H. S. Fogler, "Capacitance Effects in Porous Media," SPE Reservoir Engineering, 1207 (Nov. 1988).

Kaplan, W., Nuclear Physics, Addison-Wiley, New York (1955).

Khilar, K. C., and H.S. Fogler, "Colloidally Induced Fines Migration in Porous Media," Reviews in Chem. Eng., 4(1), 43 (1987).

Klein, O., and Y. Nishina, "Uber Die Streuung von Strahlung Durch Freie Elektronen Nach der Neuen Relativistischen Quantendynamik von Dirac," $Z$. Physic. 52, 853 (1929).

Knoll, G. F., Radiation Detection \& Measurement, Wiley, New York (1979).

Koral, K. F., and A. R. Johnston, "Estimation of Organ Depth by Gamma Ray Spectral Comparison," Phys. Med. Biol., 22(5), 988 (1974).

Lin, J. S., M. M. Chen, and B. T. Chao, "A Novel Radioactive Particle Tracking Facility for Measurement of Solids Motion in Gas Fluidized Beds," AIChE J. 31(3), 465 (1985).

Lux, I., "A Handy Method for Approximate Optimization of Splitting in Monte Carlo," Nucl. Sci. \& Eng., 83(2), 198 (1983).

McKinley, R. M., "Production Logging," SPE Reprint Series, No. 19, Soc. of Petrol. Engs., Richardson, TX (1985).

Perkins, T. K., and G. C. Johnston, "A Review of Diffusion and Dispersion in Porous Media," Pet. Trans. AIME, 228, 77 (1963).

Storm, E., and H. I. Israel, "Photon Cross Sections from $1 \mathrm{KeV}$ to 100 MeV for Elements $Z=1$ to $Z=100$," Nuclear Data Tables, A7, 565 (1970).

Manuscript received Nov. 20, 1989, and revision received Apr. 18, 1990. 